\title{
Integrative epigenomic and genomic filtering for methylation markers in hepatocellular carcinomas
}

\author{
Jing Shen ${ }^{1 *}$, Clare LeFave ${ }^{2}$, Iryna Sirosh ${ }^{1}$, Abby B. Siegel ${ }^{3}$, Benjamin Tycko ${ }^{2,4}$ and Regina M. Santella
}

\begin{abstract}
Background: Epigenome-wide studies in hepatocellular carcinoma (HCC) have identified numerous genes with aberrant DNA methylation. However, methods for triaging functional candidate genes as useful biomarkers for epidemiological study have not yet been developed.
\end{abstract}

Methods: We conducted targeted next-generation bisulfite sequencing (bis-seq) to investigate associations of DNA methylation and mRNA expression in HCC. Integrative analyses of epigenetic profiles with DNA copy number analysis were used to pinpoint functional genes regulated mainly by altered DNA methylation.

Results: Significant differences between HCC tumor and adjacent non-tumor tissue were observed for 28 bis-seq amplicons, with methylation differences varying from 12\% to 43\%. Available mRNA expression data in Oncomine were evaluated. Two candidate genes (GRASP and TSPYL5) were significantly under-expressed in HCC tumors in comparison with precursor and normal liver tissues. The expression levels in tumor tissues were, respectively, 1.828 and -0.148 , significantly lower than those in both precursor and normal liver tissue. Validations in an additional 42 paired tissues showed consistent under-expression in tumor tissue for GRASP (-7.49) and TSPYL5 (-9.71). A highly consistent DNA hypermethylation and mRNA repression pattern was obtained for both GRASP (69\%) and TSPYL5 (73\%), suggesting that their biological function is regulated by DNA methylation. Another two genes (RGS17 and NR2E1) at Chr6q showed significantly decreased DNA methylation in tumors with loss of DNA copy number compared to those without, suggesting alternative roles of DNA copy number losses and hypermethylation in the regulation of RGS17 and NR2E1.

Conclusions: These results suggest that integrative analyses of epigenomic and genomic data provide an efficient way to filter functional biomarkers for future epidemiological studies in human cancers.

\section{Background}

Epigenome-wide association studies [1-7], including ours $[8,9]$ have identified large panels of genes with aberrant DNA methylation in hepatocellular carcinoma (HCC). In two previous studies, we found an overlap of 402 significantly hypermethylated and 985 hypomethylated genes in $\mathrm{HCC}$ tumor tissues in comparison with adjacent nontumors using Illumina $27 \mathrm{~K}$ and $450 \mathrm{~K}$ methylation arrays $[8,9]$. Hypermethylation of 275 of the genes was consistent with other epigenome-wide studies [1-7], and the gene list includes several well-known tumor suppressor genes (TSGs) such as APC (adenomatous polyposis coli), $p 16 / C D K N 2 A$ (cyclin-dependent kinase inhibitor 2A) and RASSF1 (Ras association domain family member 1 ). These

\footnotetext{
* Correspondence: js2182@cumc.columbia.edu

${ }^{1}$ Department of Environmental Health Sciences, Mailman School of Public Health, Columbia University Medical Center, New York, NY 10032, USA Full list of author information is available at the end of the article
}

data indicate the reliability of genome-wide methylation results. Simultaneously, a large number of genes were identified for the first time as aberrantly methylated (127 hypermethylated and 308 hypomethylated) in HCC tumor tissue $[8,9]$ providing a resource to examine novel etiological risk factors and biologically relevant epigenetic markers for early diagnosis of HCC. Whether the aberrant methylation has functional consequences and can serve as sensitive markers of HCC is largely unknown in populationbased epidemiological studies. Moreover, there is a lack of systematic analyses that integrate DNA methylation changes with genetic/epigenetic factors (copy number variations (CNVs), microRNA (miRNA) expression and histone modification, etc.) that potentially influence a gene's biological functions. From the viewpoint of epidemiological studies alone, evidence of significant differences in DNA methylation between tumor and non-tumor tissues is insufficient to establish a causative role for the candidate genes in 
tumorigenesis. The large number of genes identified in previous epigenome-wide association studies [1-9] has complicated their application in larger population-based validation studies to determine in a cost-effective way their utility as risk factors, as well as early diagnostic/prognostic markers. Therefore, comprehensive analyses of available genetic and epigenetic data together may help us to better understand the functions of genes identified as being mainly regulated by aberrant DNA methylation, and narrow down the number of crucial methylation markers involved in hepatocarcinogenesis for future large epidemiological studies, including those which might involve using blood as an indirect surrogate, or cell-free tumor-derived DNA as a directly relevant analyte.

A limited number of $\mathrm{CpG}$ sites per gene or region are analyzed in genome-wide array-based methylation studies. Thus, we conducted further analysis by targeted nextgeneration bisulfite sequencing (bis-seq) to validate the genes identified as having the largest changes in DNA methylation based on both Illumina $27 \mathrm{~K}$ and $450 \mathrm{~K}$ array data. Our targeted bis-seq approach used PCR based amplification followed by sequencing on an Illumina MiSeq to cover multiple CpG sites (2-57 CpG sites) in candidate genes. This method allows for nucleotide level resolution and high sequencing depth and was able to cover multiple CpG sites for each of the candidate genes. To further investigate genes of interest and identify genes with inverse associations between DNA methylation and expression, we integrated the DNA methylation data with a genomic database of mRNA expression in HCC tissues and other cancer tissues, as well as cancer cell lines. Finally, we further examined the influence of genetic/epigenetic factors (such as CNVs, miRNA expression, histone modifications, etc) on gene expression to distinguish functional candidates mainly regulated by DNA methylation that may serve as promising early diagnostic markers.

\section{Methods}

\section{Study subjects}

This study was approved by the Institutional Review Board of Columbia University Medical Center. ). A waiver of consent was given because the majority of patients died before the research was carried out. Some of the living patients did give informed consent due to their interests in participating in further follow-up study. Sixty-six frozen HCC tissues from the Molecular Pathology Shared Resource of the Herbert Irving Comprehensive Cancer Center, as well as their detailed histological and clinical features including $\mathrm{HBV}$ ( $\mathrm{HBsAg}$ ) and $\mathrm{HCV}$ (anti-HCV) status were available from our prior study of methylation using Illumina arrays [9]. Twenty-four of these paired tumor/adjacent non-tumor tissues with different viral status were selected for targeted bis-seq of multiple $\mathrm{CpG}$ sites to determine methylation level, as well as for mRNA expression measured by quantitative reverse transcription PCR (qRT-PCR).

\section{Selection of candidate genes and regions}

To select candidate genes and regions for further validation of DNA methylation by targeted bis-seq, we compared the significant $\mathrm{CpG}$ sites and genes identified in our previous studies using Illumina $450 \mathrm{~K}$ and $27 \mathrm{~K}$ arrays that were deposited in NCBI's Gene Expression Omnibus (GEO) database (accession number GSE54751 and GSE37988) [8, 9]. We found an overlap of 402 hypermethylated genes (covering $505 \mathrm{CpG}$ sites) and 985 hypomethylated genes (covering 1242 CpG sites). Because previous studies using a candidate gene approach have found more hypermethylated TSGs and DNA repair genes [10-21] than hypomethylated oncogenes in HCC, we selected a total of 20 hypermethylated and eight hypomethylated genes for the current study. Each gene had a $>20 \%$ DNA methylation difference between HCC tumor and non-tumor tissues and a Bonferroni adjusted $p$ value $<0.05$. For each hypermethylated gene, at least two amplicons were designed to cover candidate CpG sites, and one amplicon was designed for hypomethylated genes. Finally, a total of 48 PCR primers pairs were used, and after sequencing, low quality sequences were removed and genome alignment carried out. Twenty-nine of the amplicons covering 20 genes were successful and analyzed in the current study.

\section{Laboratory methods}

Bisulfite treatment was performed on $1 \mu \mathrm{g}$ DNA using the Epitect kit (Qiagen) as per the instructions. The genomic locations of candidate genes and covered $\mathrm{CpG}$ sites of the amplicons for targeted bis-seq are given in Additional file 1: Table S1. Oligonucleotide primers were designed around the CpGs of interest using MethPrimer (http://www.urogene.org/cgi-bin/methprimer/methprimer.cgi) [22], and the CS1 and CS2 Fluidigm tags were added. Primers were synthesized (IDTDNA) and verification of the amplicon size was performed. The Fluidigm Access Array was performed with the KAPA HiFi 2x Uracil + polymerase. Next the adapter and barcode sequences were added following the Fluidigm protocol using the Faststart Hi Fidelity kit (Roche) and the barcoded primers (Fluidigm). Verification of product was done on an agarose gel and cleaned-up using the Agencourt AMPure XP (Beckman Coulter). Libraries were then quantified using the Kapa Library Quantification Kit (Kapabiosystems). Samples were then pooled with $30-50 \%$ PhiX (Illumina) and loaded onto the MiSeq (Illumina) for sequencing. Libraries are clustered and sequenced with 250 nucleotide paired-ends. The Fastq files generated by sequencing were trimmed for both adapters and for a quality cut off 
of 30 using Trim Galore (http://www.bioinformatics.babraham.ac.uk/projects/trim_galore/). Sequencing alignment and methylation calls were done via Bismark [23] and bowtie2 [24]. The genome used for alignment was recent human assembly GRCh37/hg19.

Total RNA, including miRNA was isolated from frozen HCC tumor and adjacent non-tumor tissues by Qiazol and RNeasy Microarray Tissue Mini Kits (Qiagen) according to the manufacturer's protocol. For mRNA expression, $1.0 \mu \mathrm{g}$ isolated RNA $(10 \mu \mathrm{L})$ was converted to cDNA using the High-Capacity cDNA Reverse Transcription Kit. After 10 times dilution of the RT products, TaqMan ${ }^{\circ}$ Gene Expression Assays (Life Technologies) were used to detect two candidate genes GRASP (general receptor for phosphoinositides 1-associated scaffold protein: Hs00699132_g1) and TSPYL5 (TSPY-like 5: Hs00603217_s1). Data were normalized by the housekeeping gene GAPDH (glyceraldehyde-3phosphate dehydrogenase: Hs02758991_g1) as recommended by a study to validate putative reference genes in HCC tissues [25]. TaqMan Low Density Arrays (TLDA, Life Technologies), covering 670 unique human mature miRNAs were used to generate genome-wide miRNA profiles that was deposited in NCBI's GEO database (accession number GSE54751) [26].

\section{Integrative analyses}

The Oncomine database (https://www.oncomine.org) $[27,28]$ that includes cancer microarray data deposited in GEO and the Stanford Microarray Database (SMD) were used to determine the differences in mRNA expression between HCC tumor and/or precursor/normal liver tissues for 20 candidate genes, as well as the wellknown TSGs APC, CDKN2A and RASSF1 [8, 9]. Fifteen publicly available datasets were selected for the integrative analyses (http://tcga-data.nci.nih.gov/tcga/) [29-42]. Details of standardized normalization techniques and statistical methods can be found on the Oncomine website $[27,28]$. The gene expression data were $\log _{2}$ transformed, median centered per array, and the standard deviation (SD) was normalized to one per array [27, 28]. Genes with significant differences in mRNA expression $(p \leq 0.05)$ and a concordant DNA methylation and mRNA expression pattern (i.e. hypermethylation with underexpression or hypomethylation with up-regulation) in liver tissues were further examined for the potential impacts of CNVs (gain or loss) using both Oncomine and our 450K methylation intensity data by DNA-Chip Analyzer (dChip) [43]. Others were excluded from further analysis. The expression levels of miRNAs (increase or decrease) that target hypermethylated genes without losses of CNVs were analyzed. The target genes of miRNAs were identified from an online resource (http://c1.accurascience.com/miRecords/). Finally, Encyclopedia of DNA Elements (ENCODE) data (https://genome.ucsc.edu/ ENCODE/) for an HCC cell line (HepG2) and another seven cancer cell lines (GM12878, H1-hESC, HSMM, HUVEC, K562, NHEK and NHLF) were incorporated with DNA methylation results from candidate genes to examine the co-operative role of histone modifications and deoxyribonuclease (DNase I) hypersensitivity on chromatin activity. Studies showed that active histone marks include histone 3 lysine 4 monomethylation (H3K4me1) typically associated with transcriptional enhancers; histone 3 lysine 4 trimethylation (H3K4me3) typically associated with promoters; and histone 3 lysine 27 acetylation (H3K27ac) typically associated with both active promoters and enhancers $[44,45]$. Histone 3 lysine 27 trimethylation (H3K27me3) acts as a repressive histone marker to epigenetically control gene transcription [46]. DNase I sensitivity is an indicator of open chromatin, and DNase I hypersensitivity sites are typical marks for active regulatory regions [47].

\section{Statistical analysis}

Paired t-tests with Bonferroni correction for multiple testing were used to compare differentially methylated genes between tumor and adjacent non-tumor tissues. A significant difference was defined as an amplicon with a Bonferronicorrected $p$-value $\leq 0.05$. Hierarchical clustering of data was performed with the significant amplicons by tissue status (tumor $v s$. adjacent non-tumor). Each gene or miRNA's expression was separately assessed for tissue differences by one-sided Student's t test (under-expression for hypermethylated genes or over-expression for hypomethylated genes). Pearson's correlation was used to analyze the relationship between DNA methylation and mRNA/miRNA expression. Chi-square test was used to analyze the impacts of CNVs (gain, no change and loss) on gene expression (under- or up-regulation). Statistical analyses were conducted using Statistical Analysis System 9.0 (SAS Institute).

\section{Results and discussion}

Clinical and pathological characteristics of HCC patients

Clinical and pathological characteristics are described in Additional file 1: Table S2. The average age at HCC diagnosis is $57.1 \pm 7.5$ years. More patients are male $(83 \%)$, Caucasian (54\%) and positive for either HBV (33.3\%) or HCV (33.3\%) or both (16.7\%). The same proportion (45.8\%) is ever smokers or alcohol drinkers. Among HCC patients, $87.5 \%$ have pathologically defined cirrhosis and $62.5 \%$ have tumors grade III or IV.

\section{Comparison of DNA methylation results from targeted bis-seq and Illumina arrays}

A total of $29 \mathrm{CpG}$ amplicons covering 20 genes (15 hyper- and 5 hypo-methylated genes) were sequenced by targeted bis-seq. The average covered $\mathrm{CpG}$ sites for each gene was 22, and ranged from 2 to 57 (Table 1). Statistically significant methylation differences between tumor 
Table 1 Targeted bis-seq data for methylation in 20 candidate genes (29 amplicons) in 24 paired HCC tumor and adjacent non-tumor tissues

\begin{tabular}{|c|c|c|c|c|c|c|}
\hline \multirow{2}{*}{$\begin{array}{l}\text { Gene } \\
\text { symbol }\end{array}$} & \multirow{2}{*}{$\begin{array}{l}\text { Amplicons } \\
\text { name }\end{array}$} & \multirow{2}{*}{$\begin{array}{l}\text { No. of covered } \\
\text { CpG sites }\end{array}$} & \multirow{2}{*}{$\begin{array}{l}\text { Non-tumor } \\
\text { Mean, SD }\end{array}$} & Tumor & \multirow[t]{2}{*}{ Difference } & \multirow{2}{*}{$\begin{array}{l}\text { Adjustec } \\
P \text { value }\end{array}$} \\
\hline & & & & Mean, SD & & \\
\hline CDKL2 & CDKL2.a & 11 & 6.49 (8.35) & $33.52(18.80)$ & 27.03 & $2.50 E-04$ \\
\hline CDKL2 & CDKL2.b & 15 & 7.59 (11.57) & $41.29(21.98)$ & 33.70 & 1.94E-04 \\
\hline CDKL2 & CDKL2.C & 27 & $13.20(13.50)$ & $37.15(20.68)$ & 23.95 & 2.83E-02 \\
\hline CLCN1 & CLCN1 & 28 & $16.31(10.83)$ & $47.84(21.93)$ & 31.53 & 3.01E-04 \\
\hline DUOX1 & DUOX1 & 36 & $16.93(16.93)$ & $36.98(24.43)$ & 20.05 & 2.77E-01 \\
\hline GRASP & GRASP.a & 24 & $12.66(8.70)$ & $42.45(23.56)$ & 29.79 & 3.92E-04 \\
\hline ILDR2 & ILDR2.a & 23 & $7.66(4.22)$ & $43.18(27.32)$ & 35.52 & 4.21E-05 \\
\hline MAST1 & MAST1.a & 16 & $16.40(20.56)$ & 59.15 (33.23) & 42.75 & 4.99E-04 \\
\hline MAST1 & MAST1.b & 2 & $10.42(10.98)$ & $34.74(21.02)$ & 24.32 & 4.33E-03 \\
\hline MAST1 & MAST1.C & 19 & $15.25(8.44)$ & $38.92(14.03)$ & 23.67 & 5.15E-05 \\
\hline$N K \times 6-2$ & NKX6-2.b & 24 & $26.17(9.56)$ & $54.97(17.70)$ & 28.80 & 4.25E-05 \\
\hline OTX1 & OTX1.a & 8 & $22.53(9.14)$ & $52.15(24.09)$ & 29.62 & 2.63E-03 \\
\hline OTX1 & OTX1.b & 28 & $17.68(12.10)$ & $49.60(17.44)$ & 31.92 & 1.85E-04 \\
\hline SERHL & SERHL.a & 16 & $11.94(4.38)$ & 35.71 (13.19) & 23.77 & 3.82E-07 \\
\hline SERHL & SERHL.b & 11 & $4.86(2.26)$ & $17.15(12.24)$ & 12.29 & $1.08 \mathrm{E}-03$ \\
\hline SPAG6 & SPAG6.b & 53 & $16.07(5.05)$ & $34.12(15.67)$ & 18.04 & $1.59 \mathrm{E}-03$ \\
\hline SPDYA & SPDYA.b & 11 & $46.93(17.40)$ & 73.91 (18.14) & 26.98 & 1.29E-02 \\
\hline TRIL & TRIL.a & 16 & $18.13(6.02)$ & $53.25(20.58)$ & 35.12 & $1.01 \mathrm{E}-05$ \\
\hline TRIL & TRIL.b & 22 & $13.88(8.85)$ & 36.95 (17.75) & 23.07 & 2.22E-03 \\
\hline TRIL & TRIL.C & 17 & $9.73(6.70)$ & $41.44(17.52)$ & 31.71 & $6.62 \mathrm{E}-06$ \\
\hline TRIL & TRIL.e & 44 & $12.10(10.22)$ & $38.52(19.07)$ & 26.42 & 1.55E-03 \\
\hline TSPYL5 & TSPYL5 & 57 & $13.83(12.12)$ & 41.21 (18.79) & 27.38 & 8.09E-04 \\
\hline USP44 & USP44.C & 9 & $61.31(10.17)$ & $73.45(10.01)$ & 12.14 & $6.81 \mathrm{E}-03$ \\
\hline ZNF397OS & ZNF397OS.a & 32 & $13.76(11.59)$ & $38.94(18.21)$ & 25.18 & $3.10 \mathrm{E}-04$ \\
\hline FAM66B & FAM66B & 13 & 91.18 (3.59) & 71.89 (17.30) & -19.29 & 9.49E-04 \\
\hline KCNQ2 & KCNQ2 & 44 & $74.06(14.65)$ & $45.21(22.68)$ & -28.85 & 3.99E-03 \\
\hline PROKR2 & PROKR2 & 14 & $83.57(14.68)$ & $65.11(12.97)$ & -18.46 & 1.11E-02 \\
\hline PTPRN2 & PTPRN2 & 8 & 74.68 (11.71) & $53.60(17.39)$ & -21.08 & $6.50 \mathrm{E}-03$ \\
\hline REXO1L2P & REXO1L2P & 12 & 84.14 (14.58) & $53.98(22.99)$ & -30.16 & 1.75E-03 \\
\hline
\end{tabular}

* All adjusted $p$ values are less than 0.05 with Bonferroni correction for multiple testing except DUOX1

and non-tumor tissues (Bonferroni corrected $p<0.05$ ) were observed for 28 amplicons with methylation differences varying from $12 \%$ to $43 \%$, including 14 hyper- and 5 hypo-methylated genes. Only one hypermethylated gene (DUOX1) showed a non-significant tumor/non-tumor difference $(p=0.277)$. This suggests that about $5 \%(1 / 20)$ of genes identified by Illumina $450 \mathrm{~K}$ array may be false positive findings even after adjustment for multiple comparison and using stringent selection criteria.

The results of targeted bis-seq are shown for GRASP (25 CpG sites) and TSPYL5 (57 CpG sites) in Additional file S1: Figure S1. Generally, within each sample, DNA methylation levels across different $\mathrm{CpG}$ sites were consistent regardless of tumor status. For hypermethylated genes, most tumor tissues displayed higher levels of DNA methylation compared to adjacent non-tumor tissues for each individual $\mathrm{CpG}$ site and the mean of all CpG sites. The direction of the methylation difference (hyper- or hypo-) between tumor and non-tumor tissues for targeted bis-seq was $100 \%$ concordant with that from $27 \mathrm{~K}$ and $450 \mathrm{~K}$ data $[8,9]$. Additional file 1: Figure S2 shows two examples of mean DNA methylation comparisons for GRASP and TSPYL 5 by targeted bis-seq and $450 \mathrm{~K}$ array approaches. Statistically significant DNA hypermethylation was observed in HCC tumor tissue compared to non-tumor tissue for both genes. These data demonstrated the accuracy and reliability of both targeted bis-seq and Illumina 27K/ $450 \mathrm{~K}$ methylation arrays. 
Comparison of mRNA expression in HCC tumor, precursors and normal liver tissues

With more and more epigenomic and genomic studies completed in tumor tissues, large panels of candidate genes are available that need further verification for their biological functions before they can be applied to populationbased molecular epidemiological studies. Comparison of mRNA expression patterns is an effective way to identify relevant functional changes and focus on crucial methylation markers involved in tumorigenesis.

A total of 15 candidate genes (CDKL2, CLCN1, DUOX1, MAST1, NKX6-2, OTX1, SPAG6, SPDYA, TRIL, USP44, ZNF397OS, GRASP, TSPYL5, KCNQ2, and PTPRN2) and three known TSGs (APC, CDKN2A and RASSF1) with available mRNA expression data in the Oncomine database (Additional file 1: Table S3) were evaluated for their biological functions in HCC tumor $(n=418)$, precursor $(n=142)$, and normal liver tissues $(n=325)$. Another five genes (ILDR2, SERHL, FAM66B, PROKR2, and REXO1L2P) were omitted from the analysis of mRNA expression due to the lack of Oncomine data. As expected, the expression levels of $A P C$ and $C D K N 2 A$ were significantly downregulated in $\mathrm{HCC}$ tumor tissues compared to both precursor and normal liver tissues (Additional file 1: Table S4) [29-32]. Overall, four candidate genes (CLCN1, DUOX1, GRASP and TSPYL5) were significantly under-expressed in HCC tumors in comparison with precursor and normal liver tissues [29-32], but inconsistent non-significant differences were also observed for CLCN1 and DUOX1 expression between HCC and precursor [29, 30, 33]. The expression levels of GRASP and TSPYL5 in HCC tumor tissues were, respectively, 1.828 and -0.148 , which were significantly lower (Fig. 1) than those in both precursor (2.210 and 1.585) and normal liver tissue (2.134 and 1.527) [29].
Because DNA methylation changes between HCC tumor and non-tumor tissues did not achieve statistical significance for DUOX1 (Table 1), down-regulation of its mRNA in tumor tissue was unlikely due to DNA methylation changes and was thus excluded from further analysis. Hypermethylated ZNF397OS was only significantly down-regulated in HCC tumor compared to precursor tissues [32], but not compared to normal liver tissue. Similarly, hypomethylated KCNQ2 displayed significant over-expression in tumor compared to precursor tissue [30]. Another four hypermethylated genes (CDKL2, MAST1, SPAG6 and TRIL) exhibited significant under-expression in tumor compared to normal liver tissue [29-33], but not precursor tissues. Hypomethylated PTPRN2 was significantly over-expressed in tumor tissue compared to normal liver tissue (Additional file 1: Table S4) [30]. The heterogeneous expression patterns of these genes in HCC tumor vs. precursor and normal liver tissues need further clarification. Four hypermethylated genes (NKX6-2, OTX1, SPDYA and USP44) did not show any significant difference in mRNA expression among tissues $[29,32]$, suggesting a minor biological role in hepatocarcinogenesis, and were excluded from further analysis. The lack of expression data on five genes (ILDR2, SERHL, $F A M 66 B, P R O K R 2$, and REXO1L2P) in HCC tissues may lead us to miss some important candidate genes; but it is unlikely to impact the functional genes identified in the current study.

\section{Integrative analyses of CNVs and miRNAs}

Integrative analyses of relevant genetic and epigenetic changes may help us to further understand important regulators and potential mechanisms associated with the biological functions of the genes that we have identified. Gains or losses of copy numbers in DNA have been

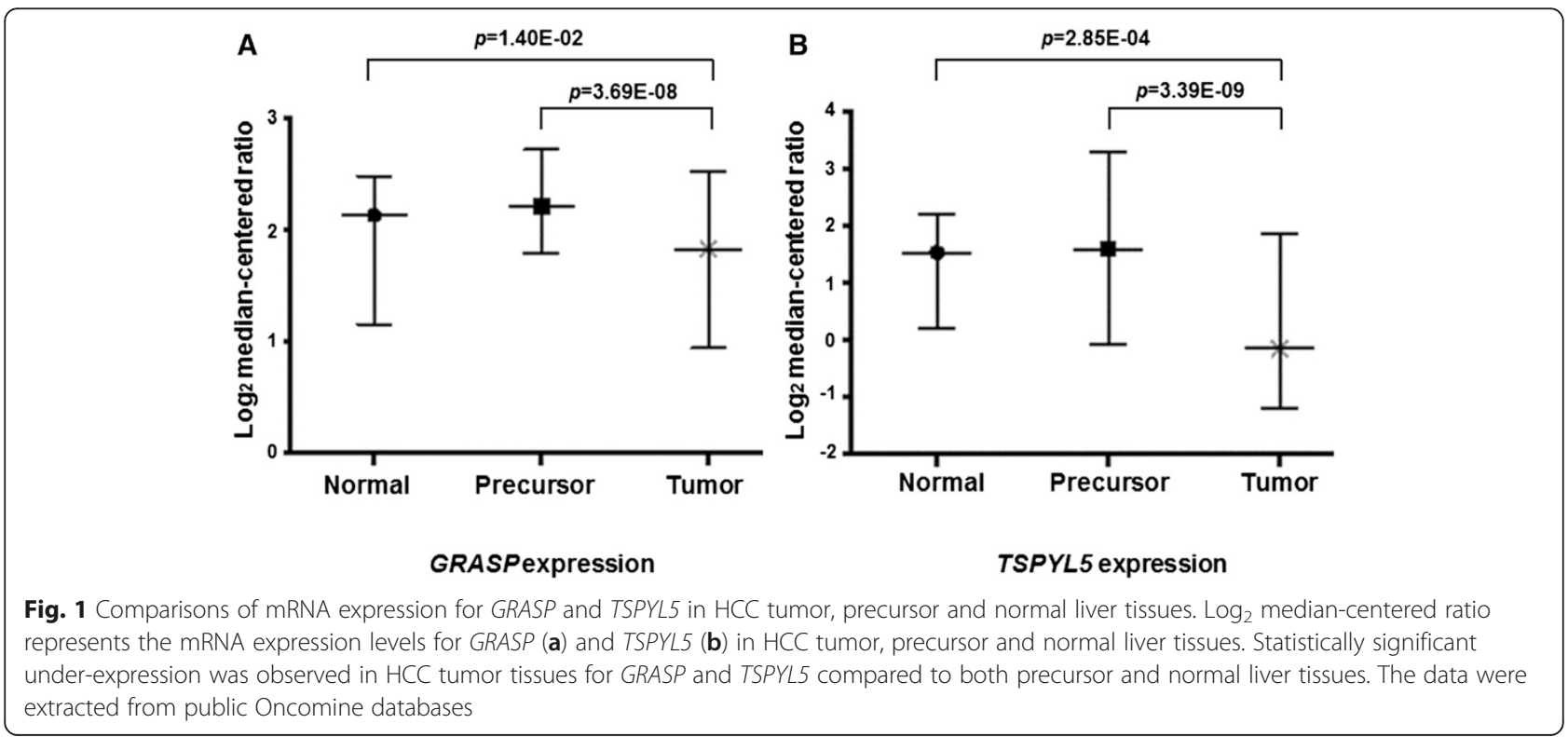




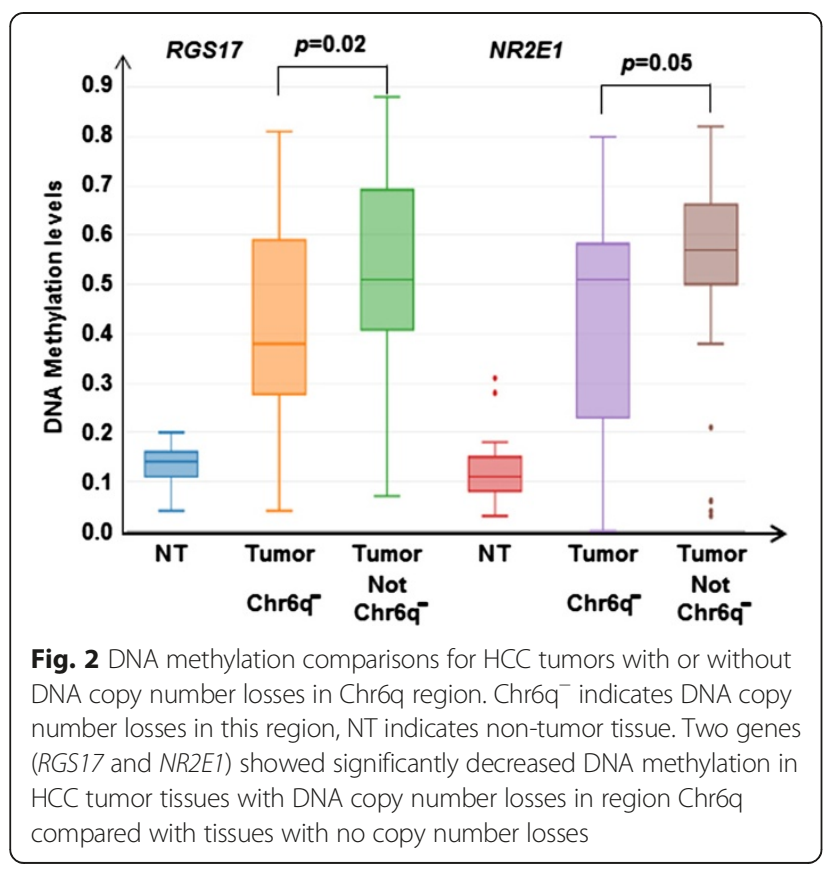

associated with relevant differences in expression of mRNAs (up- or down-regulation) [48] and play a similar biological role as DNA hypo- or hyper-methylation. Integrative analyses of CNVs was performed in $\mathrm{HCC}$ tumor $(n=209)$, precursor $(n=94)$ and normal liver tissues $(n=145)$ based on the Oncomine database (Additional file 1: Table S3), as well as in our 66 paired HCC tissues. We found no significant CNV losses for CLCN1, GRASP and TSPYL5, genes that displayed significant under-expression in HCC tumor compared to both precursor and normal liver tissues (Additional file 1: Figure S3). Similarly, no significant CNV losses were observed for MAST1, SPAG6 and TRIL, genes that showed under-expression in HCC tissue compared to normal liver tissue (data not shown).

Integrating data on copy number losses and DNA methylation changes in HCC tumor tissues, we only found two CpG sites in RGS17 (cg16924337) and NR2E1 (cg17386213) that had significantly reduced DNA methylation in tumors with loss of DNA copy number compared to those without (Fig. 2). DNA methylation levels in tumor and non-tumor tissues were, respectively 0.54 and 0.41 for RGS17 $(p=0.02)$, and 0.53 and 0.43 for NR2E1 $(p=0.05)$, suggesting a potential regulatory role for copy number losses in liver tumorigenesis in addition to DNA methylation alteration. Therefore, one allele either undergoing DNA hypermethylation or loss of copy number may lead to the partial inactivation of genes with tumor suppressive activity.

Aberrant expression of miRNAs may also regulate function of the relevant target genes. Among the six hypermethylated and under-expressed genes (CLCN1, GRASP, MAST1, SPAG6, TRIL and TSPYL5) without losses of copy number, we further examined whether miRNAs that target those genes are over-expressed in HCC tumor tissue. The numbers of miRNAs targeting each gene varied from 4 to 51 and included 1-27 detectable miRNAs in liver tissue (Additional file 1: Table S5). Overall, no significant upregulated miRNA was observed in HCC tumor tissue compared to non-tumor tissue. The expression of miR-888 targeting SPAG6 did not show a significant difference $\left(\log _{2}\right.$ fold change $=0.11, p=0.867$ ). In contrast, we found 1-8 significantly under-expressed miRNAs ( $\log _{2}$ fold changes ranging from -0.68 folds to -2.66 folds) in HCC tumors (Additional file 1: Table S6), suggesting those miRNAs were unlikely to cause the under-expression of the relevant target genes (CLCN1, GRASP, MAST1, TRIL and TSPYL5). These integrative analyses indicate that no significant CNV losses and over-expressed miRNAs that target GRASP and TSPYL5 were observed in HCC (Table 2), suggesting a minor functional impact of $\mathrm{CNVs}$ and miRNAs for these two genes.

\section{Validation of mRNAs expression for GRASP and TSPYL5}

The two promising genes (GRASP and TSPYL5) were further validated in terms of their mRNA expression in the same 24 paired HCC tissues that underwent targeted

Table 2 Comparison of significantly expressed miRNAs that target GRASP and TSPYL5

\begin{tabular}{|c|c|c|c|c|c|}
\hline \multirow{2}{*}{$\begin{array}{l}\text { Target gene } \\
\text { symbol }\end{array}$} & \multirow[t]{2}{*}{ miRNAs } & \multirow{2}{*}{$\begin{array}{l}\text { Non-tumor } \\
\text { Mean (Log2), SD }\end{array}$} & \multirow{2}{*}{$\begin{array}{l}\text { Tumor } \\
\text { Mean }(\log 2), \text { SD }\end{array}$} & \multirow{2}{*}{$\begin{array}{l}\text { Adjusted } \\
P \text { value * }\end{array}$} & \multirow{2}{*}{$\begin{array}{l}\text { Regulation in } \\
\text { HCC tumor }\end{array}$} \\
\hline & & & & & \\
\hline GRASP & hsa-miR-320b & $-8.97,0.75$ & $-10.10,0.96$ & 8.95E-03 & Down \\
\hline \multirow[t]{8}{*}{ TSPYL5 } & hsa-miR-193a-3p & $-8.21,1.06$ & $-9.53,0.88$ & 7.58E-03 & Down \\
\hline & hsa-let-7g & $-3.43,0.67$ & $-4.11,0.55$ & $2.32 \mathrm{E}-02$ & Down \\
\hline & hsa-let-7a & $-6.43,0.64$ & $-7.35,0.86$ & $1.42 \mathrm{E}-02$ & Down \\
\hline & hsa-let-7e & $-4.05,0.82$ & $-4.78,0.63$ & 3.86E-02 & Down \\
\hline & hsa-let-7c & $-6.88,0.50$ & $-8.62,1.08$ & $2.24 \mathrm{E}-04$ & Down \\
\hline & hsa-let-7b & $-4.53,0.86$ & $-5.89,1.18$ & 8.94E-03 & Down \\
\hline & hsa-miR-320b & $-8.97,0.75$ & $-10.10,0.96$ & 8.95E-03 & Down \\
\hline & hsa-miR-27b & $-5.69,0.79$ & $-6.48,0.70$ & $2.84 \mathrm{E}-02$ & Down \\
\hline
\end{tabular}

* Bonferroni correction for multiple testing 
Table $3 \log _{2}$ expression levels of two candidate mRNAs in discovery ( 24 pairs) and validation ( 42 pairs) sets by qRT-PCR assays

\begin{tabular}{|c|c|c|c|c|c|}
\hline \multirow[t]{3}{*}{ mRNAs } & \multirow[t]{3}{*}{ Samples } & \multirow{2}{*}{\multicolumn{2}{|c|}{$\begin{array}{l}\log _{2} \text { expression levels } \\
\text { Mean (SD) }\end{array}$}} & \multirow{3}{*}{$\begin{array}{l}\text { Fold } \\
\text { change }\end{array}$} & \multirow[t]{3}{*}{$P$ value } \\
\hline & & & & & \\
\hline & & Tumor & Non-tumor & & \\
\hline \multirow[t]{2}{*}{ GRASP } & 24 pairs & $-7.21(1.30)$ & $-6.51(0.89)$ & -1.62 & 2.75E-02 \\
\hline & 42 pairs & $-7.49(1.41)$ & $-6.52(1.26)$ & -1.96 & 1.53E-03 \\
\hline \multirow[t]{2}{*}{ TSPYL5 } & 24 pairs & $-9.67(1.61)$ & $-8.79(1.13)$ & -1.85 & $3.68 \mathrm{E}-02$ \\
\hline & 42 pairs & $-9.71(1.63)$ & $-8.94(1.22)$ & -1.71 & $1.25 \mathrm{E}-02$ \\
\hline
\end{tabular}

bis-seq, as well as an additional 42 paired tissues. GRASP and TSPYL5 were selected based on their consistent DNA methylation and expression patterns in tumor, precursor and normal tissues, as well as no significant influence of CNVs and miRNAs on their functions. The distribution of GRASP and TSPYL5 expression levels for each sample are shown in Additional file 1: Figure S4. A consistent and statistically significant under-expression pattern was observed for GRASP and TSPYL5 in both the 24 and 42 paired tumor tissues (Table 3). The fold-changes for GRASP and TSPYL5 were, respectively -1.62 and -1.85 in the 24 pairs, and -1.96 and -1.71 in the 42 pairs, which is consistent with the Oncomine data (Additional file 1: Table S4). When analyzing correlation of DNA methylation and expression, we found consistent hypermethylation and repression pattern for GRASP (71\% of samples) and TSPYL5 (67\%) in the 24 pairs (Additional file 1: Figure S5). In the additional 42 paired HCC tumor and adjacent non-tumor tissues, similar proportions of tumor tissues with a hypermethylation and under-expression pattern were observed for GRASP (68\%) and TSPYL5 (78\%). The correlation coefficients of DNA methylation and expression were -0.394 for GRASP $(p=0.007)$ and -0.415 for TSPYL5 $(p=0.004)$, indicating a major regulatory role for DNA methylation (Table 4). The repression of GRASP and TSPYL5 observed in tumor tissue is more likely through the mechanism of altered DNA methylation.

\section{Integrative analyses with ENCODE data}

To perform integrative analyses of DNA methylation and the ENCODE data for GRASP and TSPYL5 in HepG2 and seven other cancer cell lines, we examined the co-operative role of histone modifications and deoxyribonuclease (DNase I) hypersensitivity on chromatin activity. Around the GRASP amplicon located in the promoter region, no DNase I hypersensitivity peak was observed in HepG2 cells, indicating inactive chromatin (Fig. 3a). Further investigation of the GRASP promoter area showed that the active histone marks (H3K4me1 and H3K27ac) had no signature of upregulation in HepG2, but $\mathrm{H} 3 \mathrm{~K} 27 \mathrm{me} 3$, a repressor, displayed an increase which is consistent with the status of DNA hypermethylation in HCC tumor tissue. Similarly, neither DNase I hypersensitive sites, nor activation of histone marks (H3K4me1, H3K4me3 and H3K27ac) were observed around the TSPYL5 amplicon in HepG2 cells (Fig. 3b). No increase of H3K27me3 was observed around TSPLY5 in HepG2 cells. The different histone modifications in HepG2 and the other cell lines indicate a potentially specific role for GRASP and TSPYL5 in hepatocarcinogenesis.

GRASP is located at chromosome $12 \mathrm{q} 13.13$ and encodes a 395 amino acid protein. Identified as an all-trans retinoic acid-induced gene [49], GRASP interacts with numerous neuronal proteins of cytohesin family members Grp1 and ADP-ribosylation factor (Art) [49] to play a role in the intracellular trafficking of receptors [50-52]. GRASP has been found to be significantly hypermethylated in HBVinfected HCC tumor tissue, but changes in expression were unknown [5]. Recently, GRASP was found to be significantly hypermethylated in colorectal cancer and also negatively correlated with expression levels [53]. The

Table 4 Integrative analyses for DNA methylation, mRNA expression and CNVs in HCC tumor tissues

\begin{tabular}{lll}
\hline Variables & GRASP & TSPYL5 \\
\hline DNA hypermethylation, No (\%) & $65(98.5)$ & $64(97.0)$ \\
mRNA under-expression, No (\%) & $45(69.2)$ & $47(73.4)$ \\
$\quad$ Expression difference, Mean (SD) & $-1.63(1.44)^{*}$ & $-1.67(1.51)^{* *}$ \\
$\quad$ Methylation difference, Mean (SD) & $0.55(0.13)^{*}$ & $0.36(0.11)^{* *}$ \\
$\quad$ Copy number loss, No (\%) & $4(8.9)^{\dagger}$ & $1(2.1)^{\dagger}$ \\
$\quad$ Copy number gain, No (\%) & $14(31.1)$ & $33(70.2)$ \\
mRNA over-expression, No (\%) & $20(30.8)$ & $17(26.6)$ \\
Expression difference, Mean (SD) & $0.93(0.79)^{\#}$ & $1.12(1.38)^{\natural}$ \\
Methylation difference, Mean (SD) & $0.44(0.30)^{\#}$ & $0.39(0.07)^{\natural}$ \\
$\quad$ Copy number gain, No (\%) & $7(35.0)^{\ddagger}$ & $12(70.6)^{\ddagger}$ \\
$\quad$ Copy number loss, No (\%) & $4(30.0)$ & $0(0.0)$ \\
\hline
\end{tabular}

${ }^{*}$ Correlation coefficient $=-0.394, p=0.007 ;{ }^{* *}$ Correlation coefficient $=-0.415, p=0.004 ;{ }^{\dagger} p=0.153 ;{ }^{*}$ Correlation coefficient $=-0.199, p=0.387 ;{ }^{n}$ Correlation coefficient $=-0.032, p=0.906 ;{ }^{*} p=0.031$ 

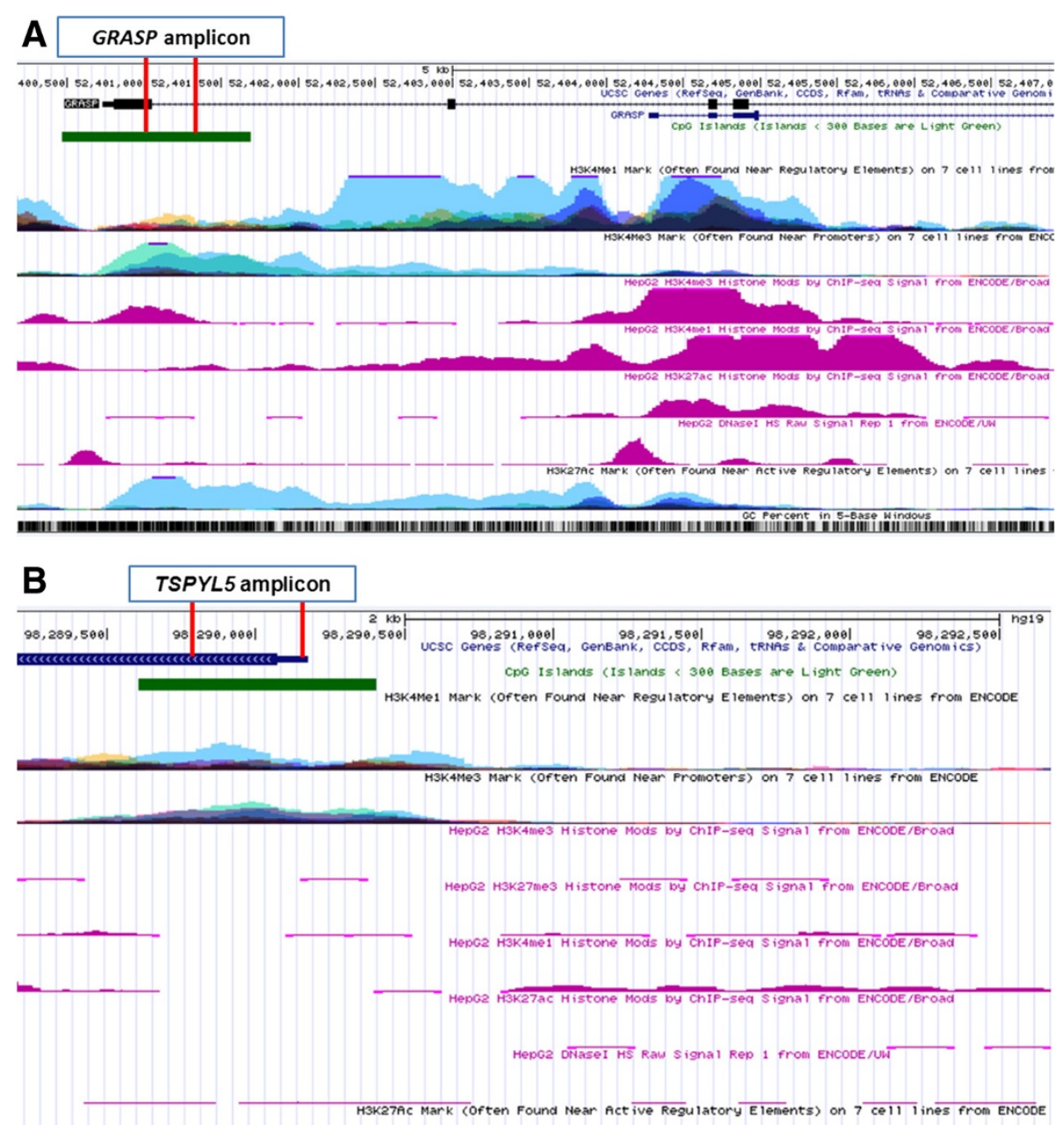

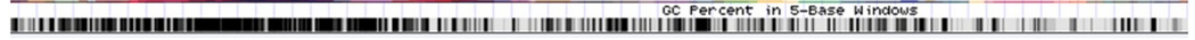

Fig. 3 UCSC genome browser tracks showing histone modifications (H3K4me1, H3K4me3, H3K27me3 and H3K27ac) and DNase I cleavage states around GRASP (a) and TSPYL5 (b) amplicons in HCC HepG2 and seven other cancer cell lines. Shown from top to bottom is the CpG island; layered H3K4Me1 activating mark, H3K4Me3 promoter specific mark in seven other cancer cell lines; H3K4Me3, H3K27me3, H3K4Me1, H3K27Ac activator marks and DNase I hypersensitive sites in HCC HepG2 cells; H3K27Ac activator in 7 other cancer cell lines; and GC percent. a: The genome browser map shows the genomic region around the GRASP (chr12:52,399,000-52,407,500). The bisulfite sequencing data covers 25 CpGs located within the promoter specific the $138^{\text {th }}$ CpG island as shown. Around the GRASP amplicon, no DNase I hypersensitivity peak was observed in HepG2 cells. Histone marks (H3K4me1 and H3K27ac) displayed no signature of active regulation in HepG2 cells, while a silencer of H3K27me3 showed a small hill. Unexpectedly, active histone H3K4me3 also exhibited a peak. The histone modifications for the seven other cancer cell lines displayed high peaks for H3K4me1, H3K4me3 and H3K27ac. b: The genome browser map shows the genomic region around TSPYL5 (chr8:98,287,500-98,292,500). The bisulfite sequencing data covers 57 CpGs located within the promoter specific the $100^{\text {th }} \mathrm{CpG}$ island as shown. Consistent with DNA hypermethylation and under-expression of mRNA, there were no active histone marks (H3K4me1, H3K4me3 and H3K27ac), as well as closed chromatin (no peak for DNase I hypersensitive sites) around the TSPYL5 amplicon in HepG2 cells, which is different from the pattern observed in seven other cancer cell lines (showing high peaks for H3K4me1, H3K4me3 and H3K27ac marks). Unexpected, no activation of H3K27me3 was observed around TSPLY5 in HepG2 cells

methylation level of GRASP is very low in non-neoplastic colorectal tissue and WBC DNA from healthy subjects, indicating potential usefulness as a non-invasive epigenetic markers [54]. It is known that $C D K N 2 A^{\text {INK4a }}$ (a tumor suppressor gene) encodes p14ARF that acts as a checkpoint within the ARF-MDM2-p53 pathway to activate and stabilize p53 [55]. When a mutagenic event occurs, the expression of p14ARF can interrupt the abnormal cell proliferation [56], while the function of GRASP is to promote ARF-Rac signaling [57]. It is biologically plausible that methylation alterations in either $C D K N 2 A^{\mathrm{INK} 4 \mathrm{a}}$ or GRASP may cause dysfunction of this pathway, and initiate tumorigenesis.

TSPYL5 is a member of the testis-specific protein Yencoded-like (TSPY-L) family of genes located on chromosome $8 \mathrm{q} 22$ and is a target of epigenetic silencing in gliomas 
including glioblastoma $[58,59]$ and gastric cancers [60]. Upon treatment with the demethylating agent 5-aza-dC in glioma [58] and gastric cancer [59] cell lines, TSPYL5 expression was restored, indicating epigenetic regulation. Stable transfection of TSPYL5 in glioma or colon cancer cell lines inhibited growth [58,60]. TSPYL5 is frequently amplified in breast cancer, and displays an oncogene-like activity. The highest level of expression was found in basallike breast cancers that correlated with shortened distant metastasis-free survival [61]. TSPYL5 can override senescence-like proliferation arrest and oncogene-induced senescence and contribute to cell transformation suggesting its role as a negative regulator of p53 function [61]. TSPYL5 protein was shown to interact with USP7 (a known deubiquitylating enzyme for p53), and suppress deubiquitination of p53 therefore marking it for degradation [61]. Moreover, the binding of TSPYL5 to USP7 can also effect other proteins targeted by USP7 for deubiquitylation including proteins involved in tumor necrosis factor alpha (TNF- $\alpha$ ) induced apoptosis [62]. We observed decreased TSPYL5 levels which would lead to an increase in p53 protein levels. In normal cells, the p53 protein is low due to rapid degradation by the proteasome through MDM2 targeted ubiquitination. Protein levels of p53 have been shown to be higher in HCC relative to adjacent normal tissue [63] but in general p53 levels were variable among HCC samples [64]. Thus, TSPYL5 hypermethylation could be a contributing factor to higher p53 protein levels in some HCC via its interaction with USP7. More studies are needed to determine the biological function of low TSPYL5 in HCC.

This integrative analyses and filtering for biologically important genes regulated by altered methylation is an important first step in the identification of biomarkers for epidemiologic studies. The novel findings of the current study are that the significant repression of GRASP and TSPYL5 in HCC tumors is likely due to epigenetic regulation, while $R G S 17$ and NR2E1 are functionally regulated by both DNA methylation and copy number losses. More importantly, many genes with aberrant DNA methylation were excluded from further evaluation because of lack of consistent evidence for their biological functions. Therefore, integrative analysis of available genetic and epigenetic data provides a high through-put and cost-effective tool to transform findings from the current study into the next step of population based epidemiological studies using non-invasive blood as a surrogate for target tissue. Integrative analysis also makes it feasible to robustly examine the tissue specificity and interpret results when evaluating the identified functional biomarkers in epidemiological studies. Because the ENCODE data does not include HCC cell lines (SNU-449, JHH2), we performed the integrative analyses on HepG2 cells derived from a human hepatoblastoma. The co-operation between DNA methylation and histone modifications on chromatin activity may be different for the different types of cell lines, and the results should be interpreted with caution.

\section{Conclusions}

The integrative analyses of epigenomic and genomic profiles provide us with an effective tool to filter biologically functional epigenetic markers for future epidemiological studies. For the first time, the expression of GRASP and TSPYL5 were identified mainly regulated by DNA methylation, while RGS17 and NR2E1 genes may be repressed by the alternative mechanisms of DNA loss of copy number or hypermethylation. With multiple layers of -omics data available (exposomics, metabolomics, phenomics, proteomics, and transcriptomics et al.), this approach will become more efficient and accurate to help epidemiologists distinguish crucial genes, pathways, epigenetic alterations and environmental factors that indicate cancer risks, development or prognosis. In addition, these biologically functional markers can also be used as preventive or therapeutic targets that enhance the efficacy of cancer control at the population level, as well as in personalized medicine by applying a molecular epidemiological study design integrated with -omics data.

\section{Availability of supporting data}

All supporting data are included as one additional file. The data sets supporting the results of this article are available in the NCBI's GEO database repository, including GSE54503 (http://www.ncbi.nlm.nih.gov/geo/query/ acc.cgi?acc=GSE54503), GSE37988 (http://www.ncbi.nlm.nih.gov/geo/query/acc.cgi?acc=GSE37988) and GSE547 51(http://www.ncbi.nlm.nih.gov/geo/query/acc.cgi?acc=G SE54751).

\section{Additional file}

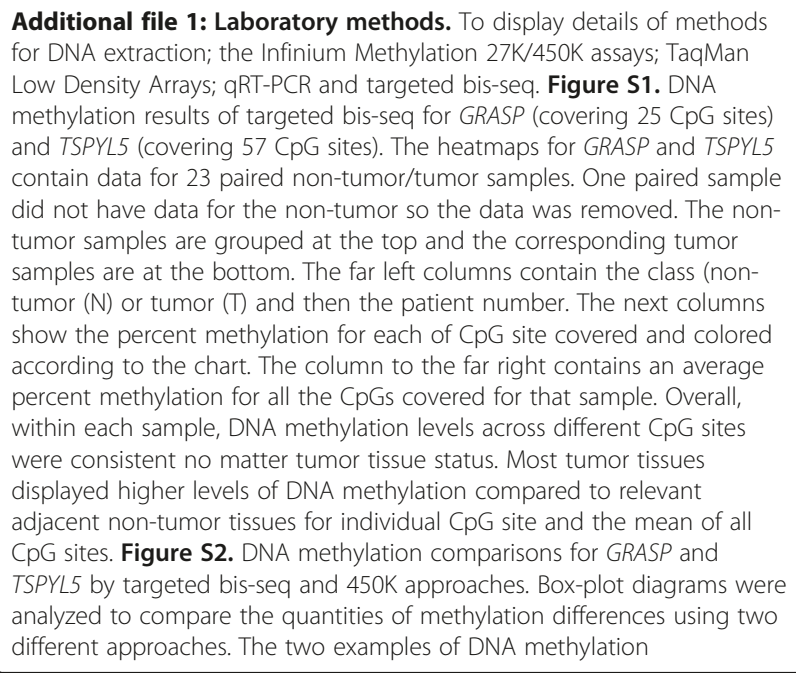


comparisons for GRASP and TSPYL5 indicate that statistically significant DNA hypermethylation was observed in HCC tumor tissue compared to non-tumor tissue. Figure S3. Gains and losses of CNV in HCC tumor, precursor and normal liver tissues for six representative genes based on the Oncomine database. Box-plot diagrams were analyzed to compare hypermethylated genes (CLCN1, GRASP and TSPYL5) under-expressed in HCC tumor tissue, and that display no significant losses of CNV compared to precursor and normal liver tissues. In contrast, statistically significant CNV losses were found in HCC tumor tissue for hypermethylated CDKL2 and ZNF397OS, and significant CNV gains were observed for hypomethylated KCNQ2 and PTPRN2, suggesting potential important role of CNVs in regulation these genes' function. Figure $\mathbf{S 4 .}$ Distribution of GRASP and TSPYL5 expression levels for each sample in 24 pairs and 42 pairs of HCC tissues. A consistent and statistically significant under-expression pattern was observed in HCC tumor tissues for GRASP and TSPYL5 in both the 24 and 42 paired samples. Figure S5. DNA methylation changes and relevant genes' (GRASP, TSPYL5) expression (Log 2 fold change) pattern in discovery (24 pairs) and validation (42 pairs) sets. DNA methylation changes (hyper-, hypo-) and relevant genes' expression (under-, over-) between individual tumor and adjacent nontumor tissues were analyzed. There were, respectively 65 and 64 HCC tumor tissues that showed significant DNA hypermethylation for GRASP and TSPYL5. Highly consistent hypermethylation and repression patterns were observed in tumor tissues for GRASP $(17 / 24,71 \%)$ and TSPYL5 (16/ $24,67 \%$ ) in the training set (Figure S5A). Validation in an additional testing set (Figure S5B) found a similar proportion of tumor tissues with hypermethylation and under-expression pattern for GRASP $(28 / 41,68 \%)$ and TSPYL5 (31/40, 78\%). However, less than one third of the tumor tissues showed a heterogeneous pattern of DNA hypermethylation and mRNA over-expression (31\% and 27\% for GRASP and TSPYL5, respectively), indicating other potential mechanisms may be involved in the regulation of expression. Table S1. Genomic locations of candidate genes and covered CpG sites of amplicons for targeted bis-seq approach. Table S2. The clinical and pathological characteristics of 24 HCC patients in the current study. Table S3. Oncomine databases for integrative gene expression and copy number variations (CNVs) analyses in liver tissues. Table S4. Representative Oncomine mRNAs expression data (Log2) to display concordant patterns with DNA methylation alterations. Table S5. Types of miRNAs that target six hypermethylated genes without losses of CNVs. Table S6. Comparison of significant expressed miRNAs that target six hypermethylated genes without losses of CNVs. Table S7.

Frequencies of copy number loss in HCC tumor by methylation status.

\section{Abbreviations}

HCC: Hepatocellular carcinoma; TSGs: Tumor suppressor genes; APC: Adenomatous polyposis coli; p16/CDKN2A: Cyclin-dependent kinase inhibitor 2A; RASSF1: Ras association domain family member 1; CNVs: Copy number variations; miRNAs: microRNAs; Bis-seq: Bisulfite sequencing; GRASP: General receptor for phosphoinositides 1-associated scaffold protein; TSPYL5: Testis-specific protein Y-encoded-like 5; ENCODE: Encyclopedia of DNA Elements; DNase I: Deoxyribonuclease; TNF-a: Tumor necrosis factor alpha; qRT-PCR: Quantitative reverse transcription PCR; TLDA: TaqMan Low Density Arrays; GEO: Gene Expression Omnibus; SMD: Stanford Microarray Database; SD: Standard deviation; H3K4me1: Histone 3 lysine 4 monomethylation; H3K4me3: Histone 3 lysine 4 trimethylation; H3K27ac: Histone 3 lysine 27 acetylation; H3K27me3: Histone 3 lysine 27 trimethylation.

\section{Competing interests}

The authors declare that they have no competing interests.

\section{Authors' contributions}

JS oversaw the study design, performed data analysis, and wrote the manuscript. $\mathrm{CL}$ ran the bis-seq assay, interpreted the results and wrote bis-seq method in the manuscript. IS run mRNA expression assay. ABS recruited the patients and oversaw the study design. BT oversaw bis-seq assay, interpreted the results and revised the manuscript. RMS oversaw the study design, interpreted the results, revised the manuscript, and obtained funding. All authors read and approved the final version of the manuscript.

\section{Acknowledgements}

This work is supported by NIH grants R01 ES005116, P30 ES009089, R03 CA156629, and pilot studies from the HICCC Epigenetics Core and NIEHS Center for Environmental Health in Northern Manhattan.

\section{Author details}

'Department of Environmental Health Sciences, Mailman School of Public Health, Columbia University Medical Center, New York, NY 10032, USA.

${ }^{2}$ Institute for Cancer Genetics, Herbert Irving Comprehensive Cancer Center, Columbia University Medical Center, New York, NY 10032, USA. ${ }^{3}$ Department of Medicine, Columbia University Medical Center, New York, NY 10032, USA. ${ }^{4}$ Department of Pathology and Cell Biology, Columbia University College of Physicians and Surgeons, New York, NY 10032, USA.

Received: 11 November 2014 Accepted: 1 June 2015

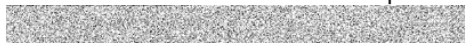

\section{References}

1. Ammerpohl O, Pratschke J, Schafmayer C, Haake A, Faber W, von Kampen $\mathrm{O}$, et al. Distinct DNA methylation patterns in cirrhotic liver and hepatocellular carcinoma. Int J Cancer. 2012;130:1319-28.

2. Neumann O, Kesselmeier M, Geffers R, Pellegrino R, Radlwimmer B, Hoffmann $\mathrm{K}$, et al. Methylome analysis and integrative profiling of human HCCs identify novel protumorigenic factors. Hepatology. 2012;56:1817-27.

3. Song MA, Tiirikainen M, Kwee S, Okimoto G, Yu H, Wong LL. Elucidating the landscape of aberrant DNA methylation in hepatocellular carcinoma. PLoS One. 2013;8:e55761.

4. Revill K, Wang T, Lachenmayer A, Kojima K, Harrington A, Li J, et al. Genome-wide methylation analysis and epigenetic unmasking identify tumor suppressor genes in hepatocellular carcinoma. Gastroenterology. 2013;145:1424-35.

5. Tao R, Li J, Xin J, Wu J, Guo J, Zhang L, et al. Methylation profile of single hepatocytes derived from hepatitis B virus-related hepatocellular carcinoma. PLoS One. 2011;6:e19862.

6. Hernandez-Vargas H, Lambert MP, Calvez-Kelm F, Gouysse G, McKay-Chopin S, Tavtigian SV, et al. Hepatocellular carcinoma displays distinct DNA methylation signatures with potential as clinical predictors. PLoS One. 2010;5:e9749.

7. Shin SH, Kim BH, Jang JJ, Suh KS, Kang GH. Identification of novel methylation markers in hepatocellular carcinoma using a methylation array. J Korean Med Sci. 2010;25:1152-9.

8. Shen J, Wang S, Zhang YJ, Kappil M, Wu HC, Kibriya MG, et al. Genome-wide DNA methylation profiles in hepatocellular carcinoma. Hepatology. 2012:55:1799-808.

9. Shen J, Wang S, Zhang YJ, Wu HC, Kibriya MG, Jasmine F, et al. Exploring genome-wide DNA methylation profiles altered in hepatocellular carcinoma using Infinium HumanMethylation 450 BeadChips. Epigenetics. 2013;8:34-43.

10. Kanai Y, Ushijima S, Hui AM, Ochiai A, Tsuda H, Sakamoto M, et al. The E-cadherin gene is silenced by $\mathrm{CpG}$ methylation in human hepatocellular carcinomas. Int J Cancer. 1997;71:355-9.

11. Matsuda Y, Ichida T, Matsuzawa J, Sugimura K, Asakura H. p16(INK4) is inactivated by extensive $\mathrm{CpG}$ methylation in human hepatocellular carcinoma. Gastroenterology. 1999;116:394-400.

12. Nishida N, Nagasaka T, Nishimura T, Ikai I, Boland CR, Goel A. Aberrant methylation of multiple tumor suppressor genes in aging liver, chronic hepatitis, and hepatocellular carcinoma. Hepatology. 2008;47:908-18.

13. Wong $\mathbb{H}$, Lo YM, Yeo W, Lau WY, Johnson PJ. Frequent p15 promoter methylation in tumor and peripheral blood from hepatocellular carcinoma patients. Clin Cancer Res. 2000;6:3516-21.

14. Yoshikawa H, Matsubara K, Qian GS, Jackson PE, Groopman JD, Manning JE, et al. SOCS-1, a negative regulator of the JAK/STAT pathway, is silenced by methylation in human hepatocellular carcinoma and shows growth-suppression activity. Nat Genet. 2001;28:29-35.

15. Yang B, Guo M, Herman JG, Clark DP. Aberrant promoter methylation profiles of tumor suppressor genes in hepatocellular carcinoma. Am J Pathol. 2003;163:1101-7.

16. Zhang YJ, Ahsan H, Chen Y, Lunn RM, Wang LY, Chen SY, et al. High frequency of promoter hypermethylation of the RASSF1A and p16 genes and its relationship to aflatoxin B1-DNA adducts level in human hepatocellular carcinoma. Mol Carcinog. 2002;35:85-92. 
17. Zhang YJ, Chen Y, Ahsan H, Lunn RM, Lee PH, Chen CJ, et al. Inactivation of the DNA repair gene 0-6-methylguanine-DNA methyltransferase by promoter hypermethylation and its relationship to aflatoxin B-1-DNA adducts and p53 mutation in hepatocellular carcinoma. Int J Cancer. 2003;103:440-4.

18. Zhang YJ, Chen Y, Ahsan H, Lunn RM, Chen SY, Lee PH, et al. Silencing of glutathione S-transferase P1 by promoter hypermethylation and its relationship to environmental chemical carcinogens in hepatocellular carcinoma. Cancer Lett. 2005;221:135-43.

19. Zhang YJ, Wu HC, Shen J, Ahsan H, Tsai WY, Yang Hl, et al. Predicting hepatocellular carcinoma by detection of aberrant promoter methylation in serum DNA. Clin Cancer Res. 2007;13:2378-84.

20. Zhong S, Tang MW, Yeo W, Liu C, Lo YM, Johnson PJ. Silencing of GSTP1 gene by $\mathrm{CpG}$ island DNA hypermethylation in HBV-associated hepatocellular carcinomas. Clin Cancer Res. 2002:8:1087-92.

21. Zhong S, Yeo W, Tang MW, Wong N, Lai PB, Johnson PJ. Intensive hypermethylation of the $\mathrm{CPG}$ island of Ras association domain family $1 \mathrm{~A}$ in hepatitis B virus-associated hepatocellular carcinomas. Clin Cancer Res. 2003;9:3376-82.

22. Li LC, Dahiya R. MethPrimer: designing primers for methylation PCRs. Bioinformatics. 2002;18:1427-31.

23. Krueger F, Andrews SR. Bismark: a flexible aligner and methylation caller for Bisulfite-Seq applications. Bioinformatics. 2011;27:1571-2.

24. Langmead B, Salzberg SL. Fast gapped-read alignment with Bowtie 2 . Nat Methods. 2012;9:357-9.

25. Cicinnati VR, Shen Q, Sotiropoulos GC, Radtke A, Gerken G, Beckebaum S. Validation of putative reference genes for gene expression studies in human hepatocellular carcinoma using real-time quantitative RT-PCR. BMC Cancer. 2008;8:350

26. Shen J, Wang A, Wang Q, Gurvich I, Siegel AB, Remotti $H$, et al. Exploration of genome-wide circulating microRNA in hepatocellular carcinoma: MiR-483-5p as a potential biomarker. Cancer Epidemiol Biomarkers Prev. 2013;22:2364-73.

27. Rhodes DR, Kalyana-Sundaram S, Tomlins SA, Mahavisno V, Kasper N, Varambally $\mathrm{R}$, et al. Molecular concepts analysis links tumors, pathways, mechanisms, and drugs. Neoplasia. 2007;9:443-54.

28. Rhodes DR, Kalyana-Sundaram S, Mahavisno V, Varambally R, Yu J, Briggs BB, et al. Oncomine 3.0: genes, pathways, and networks in a collection of 18,000 cancer gene expression profiles. Neoplasia. 2007;9:166-80.

29. Wurmbach E, Chen YB, Khitrov G, Zhang W, Roayaie S, Schwartz M, et al. Genome-wide molecular profiles of HCV-induced dysplasia and hepatocellular carcinoma. Hepatology. 2007;45:938-47.

30. Mas VR, Maluf DG, Archer KJ, Yanek K, Kong X, Kulik L, et al. Genes involved in viral carcinogenesis and tumor initiation in hepatitis $C$ virus-induced hepatocellular carcinoma. Mol Med. 2009;15:85-94.

31. Roessler S, Jia HL, Budhu A, Forgues M, Ye QH, Lee JS, et al. A unique metastasis gene signature enables prediction of tumor relapse in early-stage hepatocellular carcinoma patients. Cancer Res. 2010;70:10202-12.

32. Chen X, Cheung ST, So S, Fan ST, Barry C, Higgins J, et al. Gene expression patterns in human liver cancers. Mol Biol Cell. 2002;13:1929-39.

33. Archer KJ, Mas VR, David K, Maluf DG, Bornstein K, Fisher RA. Identifying genes for establishing a multigenic test for hepatocellular carcinoma surveillance in hepatitis C virus-positive cirrhotic patients. Cancer Epidemiol Biomarkers Prev. 2009;18:2929-32.

34. Su Al, Welsh JB, Sapinoso LM, Kern SG, Dimitrov P, Lapp H, et al. Molecular classification of human carcinomas by use of gene expression signatures. Cancer Res. 2001;61:7388-93.

35. Yu K, Ganesan K, Tan LK, Laban M, Wu J, Zhao XD, et al. A precisely regulated gene expression cassette potently modulates metastasis and survival in multiple solid cancers. PLoS Genet. 2008:4:e1000129.

36. Barretina J, Caponigro G, Stransky N, Venkatesan K, Margolin AA, Kim S, et al. The Cancer Cell Line Encyclopedia enables predictive modelling of anticancer drug sensitivity. Nature. 2012;483:603-7.

37. Garnett MJ, Edelman EJ, Heidorn SJ, Greenman CD, Dastur A, Lau KW, et al Systematic identification of genomic markers of drug sensitivity in cancer cells. Nature. 2012:483:570-5.

38. Gyorffy B, Surowiak P, Kiesslich O, Denkert C, Schafer R, Dietel M, et al. Gene expression profiling of 30 cancer cell lines predicts resistance towards 11 anticancer drugs at clinically achieved concentrations. Int J Cancer. 2006;118:1699-712.
39. Chiang DY, Villanueva A, Hoshida Y, Peix J, Newell P, Minguez B, et al. Focal gains of VEGFA and molecular classification of hepatocellular carcinoma. Cancer Res. 2008;68:6779-88.

40. Beroukhim R, Mermel CH, Porter D, Wei G, Raychaudhuri S, Donovan J, et al. The landscape of somatic copy-number alteration across human cancers. Nature. 2010;463:899-905.

41. Guichard C, Amaddeo G, Imbeaud S, Ladeiro Y, Pelletier L, Maad IB, et al. Integrated analysis of somatic mutations and focal copy-number changes identifies key genes and pathways in hepatocellular carcinoma. Nat Genet. 2012;44:694-8

42. Rothenberg SM, Mohapatra G, Rivera MN, Winokur D, Greninger P, Nitta M, et al. A genome-wide screen for microdeletions reveals disruption of polarity complex genes in diverse human cancers. Cancer Res. 2010;70:2158-64.

43. Lin M, Wei LJ, Sellers WR, Lieberfarb M, Wong WH, Li C. dChipSNP. significance curve and clustering of SNP-array-based loss-of-heterozygosity data. Bioinformatics. 2004;20:1233-40.

44. Barski A, Cuddapah S, Cui K, Roh TY, Schones DE, Wang Z, et al. Highresolution profiling of histone methylations in the human genome. Cell. 2007;129:823-37.

45. Heintzman ND, Hon GC, Hawkins RD, Kheradpour P, Stark A, Harp LF, et al. Histone modifications at human enhancers reflect global cell-type-specific gene expression. Nature. 2009;459:108-12.

46. Au SL, Ng IO, Wong CM. Epigenetic dysregulation in hepatocellular carcinoma: focus on polycomb group proteins. Front Med. 2013;7:231-41.

47. Boyle AP, Davis S, Shulha HP, Meltzer P, Margulies EH, Weng Z, et al. High-resolution mapping and characterization of open chromatin across the genome. Cell. 2008;132:311-22.

48. Kresse SH, Rydbeck H, Skarn M, Namlos HM, Barragan-Polania AH, Cleton-Jansen AM, et al. Integrative analysis reveals relationships of genetic and epigenetic alterations in osteosarcoma. PLoS One. 2012;7:e48262.

49. Nevrivy DJ, Peterson VJ, Avram D, Ishmael JE, Hansen SG, Dowell P, et al. Interaction of GRASP, a protein encoded by a novel retinoic acid-induced gene, with members of the cytohesin family of guanine nucleotide exchange factors. J Biol Chem. 2000;275:16827-36.

50. Esteban PF, Yoon HY, Becker J, Dorsey SG, Caprari P, Palko ME, et al. A kinase-deficient TrkC receptor isoform activates Arf6-Rac1 signaling through the scaffold protein tamalin. J Cell Biol. 2006;173:291-9.

51. Kitano J, Kimura K, Yamazaki Y, Soda T, Shigemoto R, Nakajima Y, et al. Tamalin, a PDZ domain-containing protein, links a protein complex formation of group 1 metabotropic glutamate receptors and the guanine nucleotide exchange factor cytohesins. J Neurosci. 2002;22:1280-9.

52. Venkataraman A, Nevrivy DJ, Filtz TM, Leid M. Grp1-associated scaffold protein (GRASP) is a regulator of the ADP ribosylation factor 6 (Arf6)dependent membrane trafficking pathway. Cell Biol Int. 2012;36:1115-28.

53. Beggs AD, Jones $A$, El Bahwary M, Abulafi M, Hodgson SV, Tomlinson IP. Whole-genome methylation analysis of benign and malignant colorectal tumours. J Pathol. 2013;229:697-704.

54. Mitchell SM, Ross JP, Drew HR, Ho T, Brown GS, Saunders NF, et al. A panel of genes methylated with high frequency in colorectal cancer. BMC Cancer. 2014;14:54.

55. Sherr CJ, Weber JD. The ARF/p53 pathway. Curr Opin Genet Dev. 2000;10:94-9.

56. Pomerantz J, Schreiber-Agus N, Liegeois NJ, Silverman A, Alland L, Chin L, et al. The Ink4a tumor suppressor gene product, p19Arf, interacts with MDM2 and neutralizes MDM2's inhibition of p53. Cell. 1998;92:713-23.

57. White DT, McShea KM, Attar MA, Santy LC. GRASP and IPCEF promote ARF-to-Rac signaling and cell migration by coordinating the association of ARNO/cytohesin 2 with Dock180. Mol Biol Cell. 2010;21:562-71.

58. Kim TY, Zhong S, Fields CR, Kim JH, Robertson KD. Epigenomic profiling reveals novel and frequent targets of aberrant DNA methylation-mediated silencing in malignant glioma. Cancer Res. 2006;66:7490-501.

59. Etcheverry A, Aubry M, de Tayrac M, Vauleon E, Boniface R, Guenot F, et al DNA methylation in glioblastoma: impact on gene expression and clinical outcome. BMC Genomics. 2010;11:701.

60. Jung Y, Park J, Bang YJ, Kim TY. Gene silencing of TSPYL5 mediated by aberrant promoter methylation in gastric cancers. Lab Invest. 2008;88:153-60.

61. Epping MT, Meijer LA, Krijgsman O, Bos JL, Pandolfi PP, Bernards R. TSPYL5 suppresses p53 levels and function by physical interaction with USP7. Nat Cell Biol. 2011:13:102-8. 
62. Zaman MM, Nomura T, Takagi T, Okamura T, Jin W, Shinagawa T, et al. Ubiquitination-deubiquitination by the TRIM27-USP7 complex regulates tumor necrosis factor alpha-induced apoptosis. Mol Cell Biol. 2013;33:4971-84

63. Zhang MF, Zhang ZY, Fu J, Yang YF, Yun JP. Correlation between expression of p53, p21/WAF1, and MDM2 proteins and their prognostic significance in primary hepatocellular carcinoma. J Transl Med. 2009;7:110.

64. Mann CD, Neal CP, Garcea G, Manson MM, Dennison AR, Berry DP.

Prognostic molecular markers in hepatocellular carcinoma: a systematic review. Eur J Cancer. 2007;43:979-92.

\section{Submit your next manuscript to BioMed Central} and take full advantage of:

- Convenient online submission

- Thorough peer review

- No space constraints or color figure charges

- Immediate publication on acceptance

- Inclusion in PubMed, CAS, Scopus and Google Scholar

- Research which is freely available for redistribution 